\title{
20
}

\section{Simulation-Aided Re-Organization of the Personnel Assignment in Dynamic Production Systems}

Gert Zülch, Jochen Heel, Jan Krüger

ifab - Institute of Human and Industrial Engineering, University of Karlsruhe, Kaiserstraße 12, D-76128 Karlsruhe, Germany Phone: +49 721/608-4250; Fax: +49 721/6944557 e-mail: heel@ifabn2.mach.uni-karlsruhe.de

\begin{abstract}
Market dynamics call for a high degree of adaptability on the side of the companies. Reacting quickly and in a flexible way to changes of customers requirements is a decisive performance indicator. With these changes it becomes more and more important to employ cost intensive personnel resources in the most effective way. Even in the case of a newly developed production system the determination of the future personnel structure is a complex planning problem. Re-organization of personnel structures needs to consider all vocational restrictions and personal preferences of the employed persons. In order to solve this complex planning problem, an approach for systematic re-organization of personnel structures was developed. This method is able to adapt personnel structures to the continuously changing general conditions by observing economical and logistical goal achievements.
\end{abstract}

Keywords

Personnel Assignment, Personnel-Oriented Simulation, Work Organization, Reorganization of Organizational and Personnel Structures, Approach for Systematic Re-organization of Personnel Structures 


\section{INTRODUCTION}

\subsection{Need for Permanent Re-organization of Personnel Structures}

Enterprises must adapt to the continuously changing market conditions in order to achieve long-term competitiveness. Therefore, enterprises cannot persist on maintaining existing organizational structures because these structures are only able to deal with changing production situations in a limited way (see Goldmann, Nagel, Preiss 1996, p. 3). Thus, it becomes more and more important to apply adequate re-organizational measures to the cost intensive personnel resources in the most effective way (following Ehlers 1997, p. 114).

This situation applies also to the "Extended Enterprise", which offers a new concept to manage these changing conditions. "Agility" and "virtuality" are important keywords in this context. The concept of the "Extended Enterprise" is described by short-cycled re-organizational measures, resulting in high adaptability of enterprises (Warnecke 1996, pp. 327). The consequence concerning staff is the need of permanent re-assignment of personnel structures in these operational units, which have been planned and configured for short-term use exclusively. Suitable and effective planning tools are needed to solve such complex and dynamic planning problems in a quickly and flexible way.

\subsection{Re-organization of Personnel Assignment}

Due to permanently changing requirements in production systems, existing personnel structures have to be adjusted to the relevant requirement situation. In general, personnel structures can be adjusted varying the number of employed persons or their abilities.

The re-organization of personnel structures is characterized by the need to consider all personnel abilities (e.g. affections, talents) and all vocational restrictions (e.g. assignment restrictions caused by the age distribution) of the employed persons. The aim is to find an optimal combination between the existing and an improved organizational structure for each production unit by minimizing costs and maximizing logistical goal achievements.

\section{METHOD FOR THE SYSTEMATIC RE-ORGANIZATION OF PERSONNEL STRUCTURES}

Personnel structuring problems can be characterized by (a) a large number of ways to assign a person to a job and (b) the conflicting logistical and monetary goals. Simulation aided approaches are the most effective way for planning personnel structures (Heel, Heitz 1995, S. 350). Typically, simulation aided planning of personnel structures is achieved by using the usual trial-and-error 
approach. With this approach, good personnel structures may only be found by chance, resulting in high effort. Therefore, Heitz (1994) developed a two-phasesapproach with heuristic search strategies, which allows for systematically planning personnel structures. The method combines search strategies and simulation techniques.

The two-phases-approach is based on the Great Deluge Algorithm (Dueck 1993), a heuristic search strategy. The method was originally able to plan personnel structures only in the case of newly developed production systems (Heitz 1994; Zülch 1996). However, to use this approach for the systematic reorganization of existing personnel structures several modifications and improvements are needed.

\subsection{Description of Planning Method}

Technical production systems, built out of given operating equipment (machines and manual workplaces), as well as the production program represented by a set of orders for various products in different numbers, are fixed input data for the personnel structure problem. The improvement procedure starts with the definition and the modeling of the planning problem, using the simulation tool ESPE (Engpaßorientierte Simulation von Personalstrukturen). ESPE is a personnel-oriented simulation tool specially developed to solve personnel structuring problems.

The procedure of finding a solution is divided into two steps (Figure 1).

Originally, in the first step (initialization phase) several starting solutions for personnel structures are generated using different heuristic algorithms. In the case of re-organization of personnel structures the existing personnel structure represents the initial situation for the improvement procedure. Modeling the existing personnel structure encloses modeling vocational restrictions and personal preferences (see Chapter 2.2).

In the second step (iteration phase), the modeled personnel structure is improved through simulation by using ex ante defined strategies. Evaluation of the simulated personnel structure considers the average lead time, the system output and the average personnel load as logistical goals, and the statically calculated personnel costs and also the personnel adjustment costs as financial goals. 


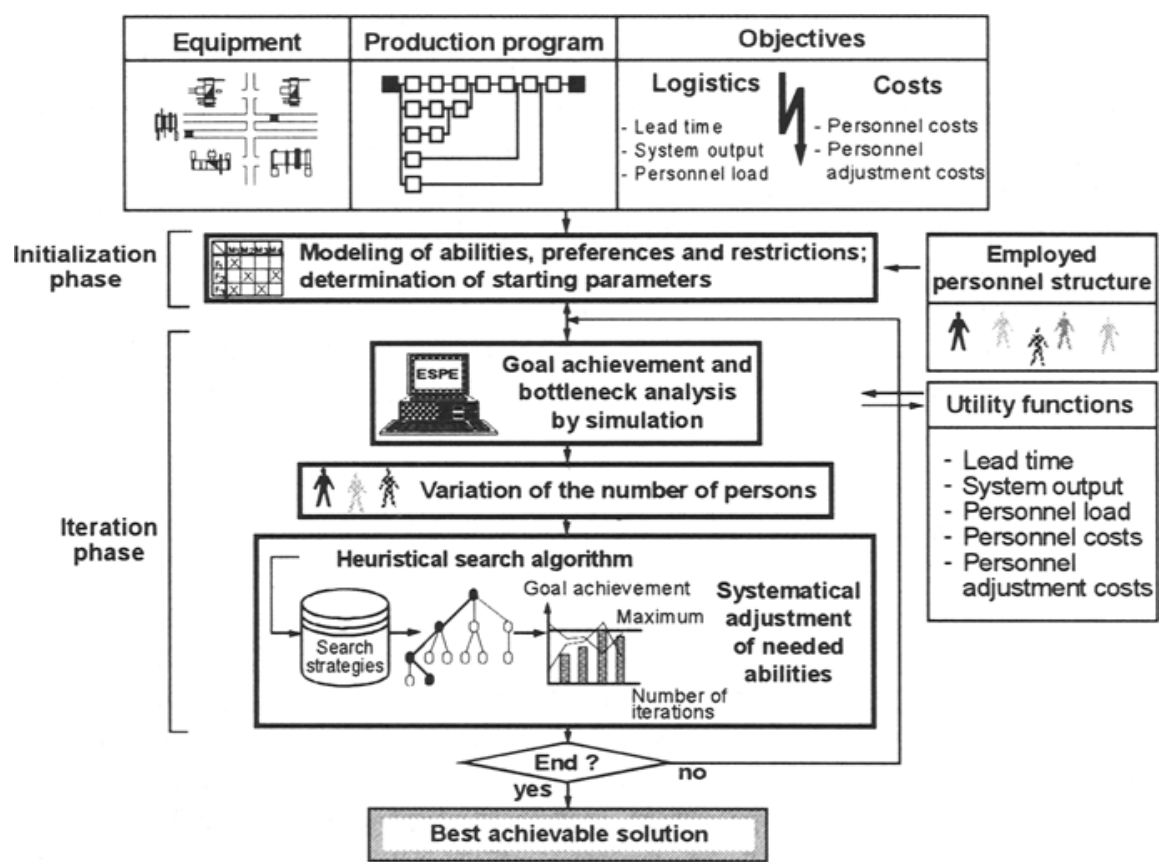

Figure 1 Approach for systematic re-organization of personnel structures.

\subsection{Modeling of Personal Preferences and Ability Restrictions}

One of the significant input masks of the simulation tool ESPE is the functionequipment-matrix, where personnel abilities of workers can be modeled easily and without modeling limits (Figure 2). The function-equipment-matrix of a technical production system contains all function elements appearing in the production program. The ability of a worker is defined by the set of all function elements he can execute in a production system. A function element is interpreted as an ability (e.g. milling), which is to be executed on a certain machine (e.g. milling machine 231; Heitz 1994, p. 30 f.). 


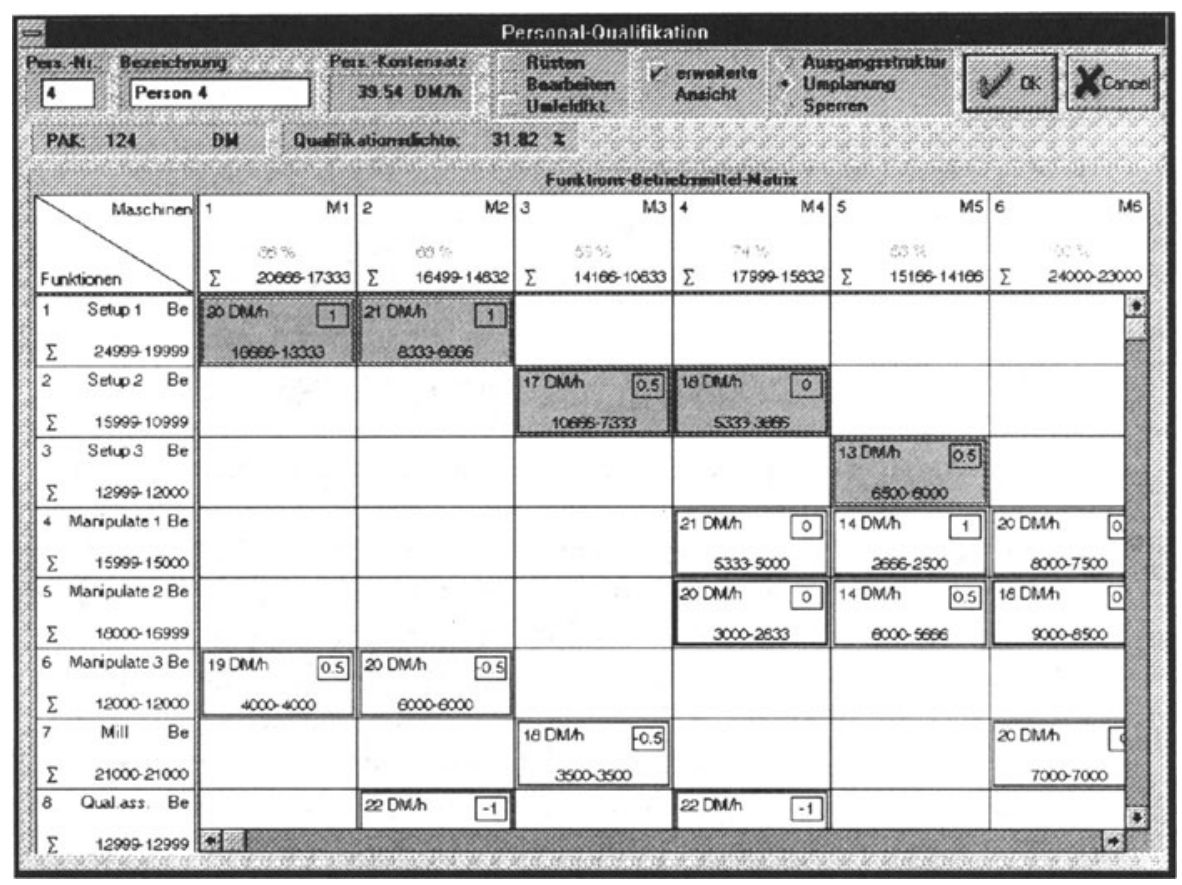

Figure 2 Modeling of abilities using the simulation tool ESPE.

However, an approach for systematic re-organization of personnel structures requires further modeling possibilities. Therefore, the simulation tool ESPE has been extended. At first, a method had to be implemented which allowed the "blocking" of function elements. This means blocked function elements can not be assigned or reduced during the improvement procedure using the strategies for calculated adjustment (Chapter 2.3). Moreover, for each function element an assignment priority can be fixed. The assignment priority is defined as a priority value in the closed interval $[-1 ; 1]$.

If the priority value of a person for a certain function element has a high positive value, the function element will be assigned preferentially to this person during the improvement procedure. A highly negative priority value for a certain function element implies that the function element will be reduced preferentially from this person during the improvement procedure. Besides the priority value as a statical input data for the assignment, the modification of the abilities is influenced by taking dynamical factors into consideration, depending on the results of the simulation run (Chapter 2.3). The determination of the priority value can be done by interviewing workers or supervisors (e.g. craftsmen).

After completing the improvement procedure, the simulation tool ESPE enables the planer to read out directly the needed abilities for each person and the per- 
sonnel recruiting requirement, can be defined, or the personnel layoff requirement, respectively.

\subsection{Determination of Assignment Values for Ability Enlargement and Simplification}

Basically, strategies for calculated adjustment of the needed abilities can be divided into:

- strategies for calculated job enlargement (if the achievement of the logistical goals is not sufficient) and

- strategies for calculated job simplification (if personnel costs are too high).

The adjustment of the personnel structure to changing conditions is realized iteratively by an improvement procedure. Special simulation key data are needed to determine the prospective workers of which the ability should be changed. Hence, for each person $p$ and separately for each function element $k$, an assignment value $A V A_{p, k}$ is calculated as the sum of weighted influence impacts based on simulation data and further statically calculated data. The assignment value $A V A_{p, k}$ serves as a prediction value to achieve a balance between smoothly running production and low personnel costs. The weighting of the influence impacts can be determined by the planner depending on the planning situation and objectives.

Decisive for the systematic enlargement, i.e. reduction of certain function elements from a certain person, is the appearance of abilities bottlenecks in a simulation run. To investigate these bottlenecks in a simulation run, the number of events when abilities are requested in vain is a useful indicator. In the case of job enlargement, such function elements will be assigned preferrably to persons which are often requested in vain, so as to avoid these bottlenecks in future simulation runs. Contrarily, in the case of job simplification such function elements will be preferrably reduced from persons who are no potential bottlenecks in the simulation run.

In order to investigate a person whose abilities should be modified, the potential availability of persons during a futile request is recorded by the simulation tool. In addition to the potential availability of persons during a futile request, the average personnel load, the wage rates of the persons, and the assignment priority for the function elements are also considered for calculating the assignment value $A V A_{p, k}$.

The assignment value $A V A_{p, k}$ is subdivided into an assignment value $A V E_{p, k}$ for job enlargement and an assignment value $A V R_{p, k}$ for job simplification according to different calculation instructions. In case of job enlargement, a certain percentage (e.g. $20 \%$ ) of abilities requested most often in vain are assigned to a certain percentage (e.g. $25 \%$ ) of the persons possessing the highest assignment value $A V E_{p, k}$ to get rid of some of the bottlenecks. In the case of job simplification, a certain percentage (e.g. $40 \%$ ) of function elements requested most rarely in vain 
are reduced from a certain percentage (e.g. $50 \%$ ) of the persons possessing the highest negative assignment value $A V R_{p, k}$ in order to avoid new bottlenecks.

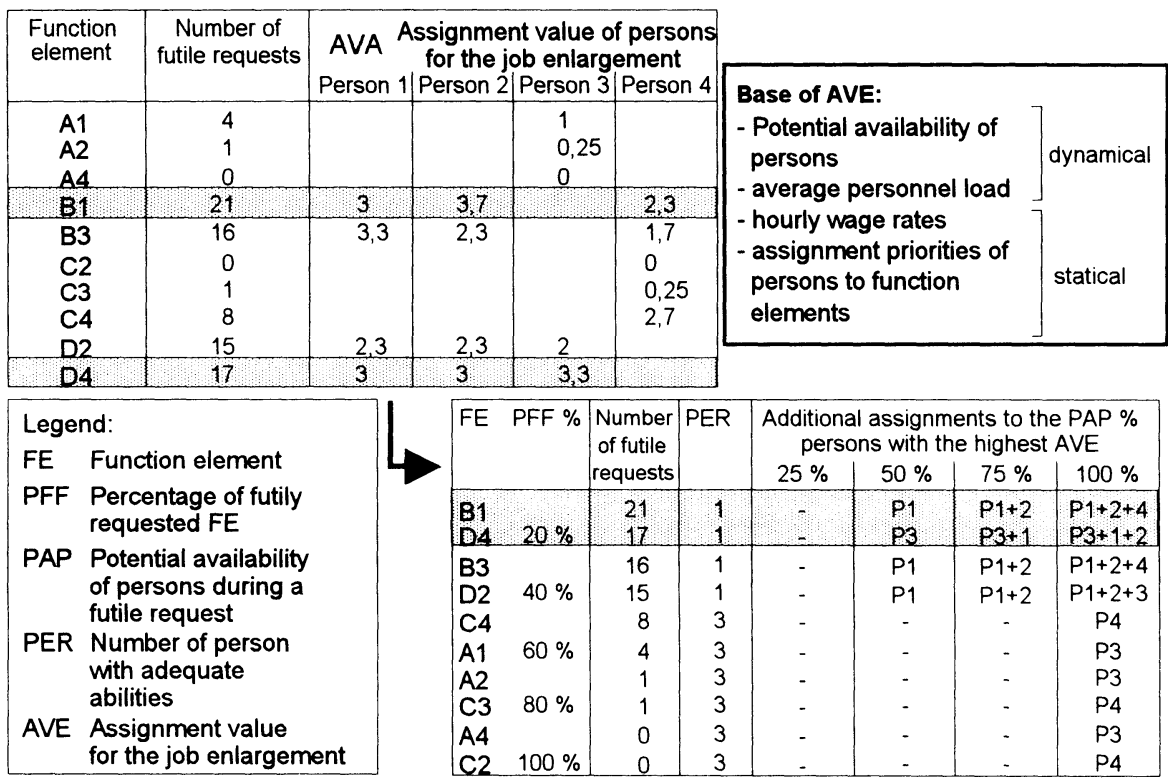

Figure 3 Strategy for the calculated enlargement of personnel abilities.

In addition to planning of a future personnel structure concerning abilities, the re-organization method is also able to systematically plan the number of required persons. The basic idea is to introduce tolerable low and high limits for average personnel loads and a so-called abilitiy density. The average personnel load is an indicator for the necessity of modifying the number of persons causing an overcharge or under-utilization of personnel capacities. Basically, the ability density of a personnel structure is the proportion of the number of function elements a person can carry out in a production system compared to the maximum possible number of function elements in this production system. For example, an ability density of $100 \%$ expresses that each person can carry out each function on each machine (universalist). Therefore, the low and high limits for the qualification density serve to avoid that the personnel structure consists only of universalists or specialists. A specialist is a person who can carry out only one function in a production system.

For example, by overstepping a certain average personnel load (e.g. $75 \%$ ) or a certain ability density (e.g. $80 \%$ ) the number of persons will be increased. This means that an additional person is assigned to the production unit. For the additional person the needed ability is found in the further iteration steps of the improvement procedure. When remaining below a certain average personnel load (e.g. $55 \%$ ) or a certain ability density (e.g. $35 \%$ ), the number of persons will be 
reduced. In the course of the improvement procedure the Great Deluge Algorithm can cancel each job enlargement or simplification step if it is necessary to achieve better goal achievements.

\subsection{Determination of Personnel Adjustment Costs}

Furthermore, the evaluation basis for the decision between several planning solutions is enlarged by personnel adjustment costs. The personnel adjustment costs are composed of the costs of personnel recruiting (e.g. advertising expense), the costs for the personnel layoff (e.g. redundancy pay) and the costs for the modification of abilities in the production unit (e.g. costs for a training program). The personnel costs are calculated depending on the required function elements for each person separately, as well as accumulated for the whole personnel structure.

\section{RE-ORGANIZATION OF PERSONNEL STRUCTURES IN A PRODUCTION ISLAND}

The effectiveness of the newly developed procedure for the systematic reorganization of personnel structures is demonstrated in a case study of a specific production island for gear-box manufacturing. In the initial situation, a production program composing of 12 different production orders is going to be manufactured on 16 machines in variable lot-sizes. The existing personnel structure consists of 12 persons with different abilities. Furthermore, certain persons are not suitable for further training measurements. At the beginning of the planning measurement, the personal preferences and the vocational restrictions are established by interviewing the persons and their supervisors.

Due to raising production requirements accompanied by a more critical schedule situation, the number of products to be manufactured in the examined production island is going to be increased in the future. In the initial situation an average lead time degree of $88 \%$, a system output degree of $92 \%$ and an average personnel load degree of $85 \%$ is reached (for the definitions of these objectives refer to Zülch, Grobel, Jonsson 1995). The wage rates of employed persons amounts to $254 \mathrm{CU} / \mathrm{h}$ (Currency Units per hour) in total. Simulation of the changed situation with the initial personnel structure leads to a collapse of the average lead time degree (38\%) and the degree of the average system output (45\%) while raising the degree of average personnel load to $100 \%$. The equipment is loaded on average with $91 \%$. The results of the simulation run emphasizes the need for adjustment of this personnel structure.

This initial personnel structure was improved by adjusting to the changing situation using the re-organization approach in 25 iteration steps. Certain abilities and determined personal preferences, as well as restrictions, are modeled in detail using the simulation tool ESPE. The best solution derived from the improvement procedure presents an average lead time degree of $94 \%$, a system output degree of 
$92 \%$ and an average personnel load degree of $89 \%$ (Figure 4 ). The total personnel costs increase to $303 \mathrm{CU} / \mathrm{h}$. But this increase of the personnel costs can be justified by the improvement of the logisitcal goal achievements.

Thus, the presented approach for systematic re-organization of personnel structures is capable of adjusting the initial personnel structure to the changing production situation reaching very good goal achievements degrees. The result of the re-organization approach is the need for one additional person. This requirement can be satisfied by regrouping another production island. The abilities of the additional person are mainly concentrated to function elements concerning operation processing. Six persons have to be trained on an intermediate term on different function elements and for two persons a restriction of the competence can be recommended to reduce the personnel costs. The total personnel adjustment costs amount to $1252 \mathrm{CU}$. For example, the needed ability of person 4 can be read out from his function equipement matrix set off by the dark function elements (figure 2).

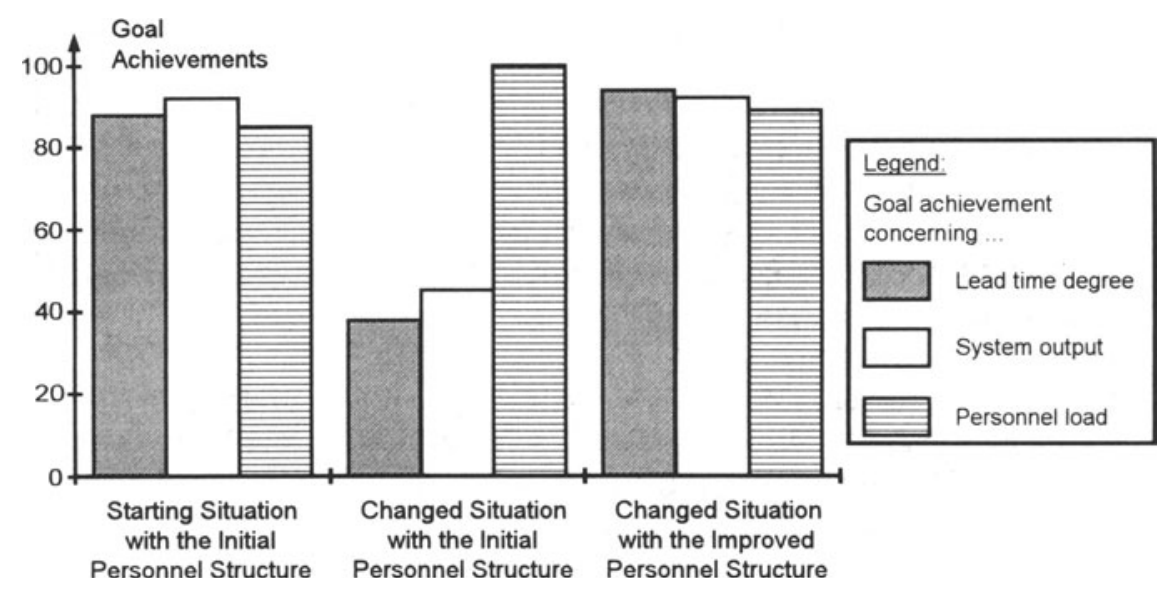

Figure 4 Comparison of the goal achievements.

\section{CONCLUSION}

The presented approach for systematic re-organization of personnel structures is a further development of the two-phases-approach with heuristic search strategies. It allows for the calculated re-organization of personnel structures. The specific feature of this approach is the adjustment to changing conditions considering all personal preferences and ability restrictions of the existing personnel structures. The improvement procedure does not only approach the adjustment of abilities, but it also approaches the most effective number of workers simultaneously. As a 
result of the improvement procedure, the planner is directly able to read out the needed abilities for each person, the personnel recruiting and the layoff requirements for the relevant production unit. In addition to conventional evaluation of personnel structures the decisional base for the planer is enlarged by allocation of personnel adjustment costs for each planning solution.

\section{REFERENCES}

Dueck, Gunter (1993) New Optimization Heuristics. The Great Deluge Algorithm and the Record-to-Record Travel. Journal of Computational Physics, 104, 86-92.

Ehlers, Jörg D. (1997) Die dynamische Produktion. Teubner, Stuttgart.

Goldmann, Steven L.; Nagel, Roger N.; Preiss, Kenneth (1996) Konfrontation mit Wandel und Instabilität, in Agil im Wettbewerb (ed. Goldmann, Steven L.; Nagel, Roger N.; Preiss, Kenneth; Warnecke, Hans-Jürgen), Springer, Berlin et al.

Heel, Jochen; Heitz, Max-Jürgen (1995) Engpaßorientierte Suchheuristik zur Personaleinsatzplanung, in Innovative Produktentwicklung und Produktionssystemplanung (eds. Grabowski, H.; Rude, S.; Zülch, G.), Veröffentlichungen des Sonderforschungsbereiches 346, Vol. 1, Universität Karlsruhe, Karlsruhe.

Heitz, Max-Jürgen (1994) Ein engpaßorientierter Ansatz zur simulationsunterstützten Planung von Personalstrukturen. ifab-Forschungsberichte aus dem Institut für Arbeitswissenschaft und Betriebsorganisation der Universität Karlsruhe, Vol. 7, Karlsruhe.

Warnecke, Hans-Jürgen (1996) Wege durchs turbulente Umfeld, in Agil im Wettbewerb.(eds. Goldmann, Steven L.; Nagel, Roger N.; Preiss, Kenneth; Warnecke, Hans-Jürgen), Springer, Berlin et al.

Zülch, Gert (1996) A Heuristic Solution to the Personnel Structure Problem, in ORBEL10, Proceedings of the Tenth Conference on Quantitative Methods for Decision Making (ed. Janssens, Gerrit K.), Belgian Operations Research Society, Brussels.

Zülch, Gert; Grobel, Thomas; Jonsson, Uwe (1995) Indicators for the Evaluation of Organizational Performance, in Benchmarking - Theory and Practice (eds. Rolstadås, Asbjørn), Chapman \& Hall, London et al. 


\section{BIOGRAPHY}

Gert Zülch: Prof. Dr.-Ing. Dipl.-Wirtsch.-Ing., born in 1946, studied mechanical engineering at the Technical University of Brunswick, Germany, and industrial engineering at the University of Technology at Aix-la-Chapelle, Germany. After his graduation as a doctor in 1979 and 10 years of experience in research and industry, in 1985 he became head of the newly founded ifab-Institute of Human and Industrial Engineering at the University of Karlsruhe, Germany.

Jochen Heel: Dipl.-Ing., born in 1967, studied mechanical engineering with the field of production technology at the University of Karlsruhe, Germany. Since 1994 he is working at the ifab-Institute as a research assistant in the field of planning and simulation of work organization.

Jan Krüger: Dipl.-Ing., born in 1967, studied mechanical engineering with the field of technical business management at the University of Dortmund, Germany. Since 1994 he is working at the ifab-Institute as a research assistant in the field of personnel-oriented modeling and simulation. 